\title{
Targeted knockout of TNF- $\alpha$ by injection of lentivirus-mediated siRNA into the subacromial bursa for the treatment of subacromial bursitis in rats
}

\author{
YI WANG, QUAN LI, XIANZHAO WEI, JIE XU, QI CHEN, SHUANG SONG, ZHE LU and ZIMIN WANG \\ Department of Orthopaedics, Changhai Hospital, Second Military Medical University, \\ Shanghai 200433, P.R. China
}

Received May 28, 2014; Accepted February 20, 2015

DOI: $10.3892 / \mathrm{mmr} .2015 .3985$

\begin{abstract}
Subacromial bursitis (SAB) is the major source of pain in rotator cuff disease. Although multiple investigations have provided support for the role of inflammatory cytokines in SAB, few have focussed on the use these cytokines in the treatment of SAB. The aim of the present study was to observe the therapeutic efficacy of lentivirus-mediated RNA interference (RNAi) on carrageenan-induced SAB by injecting lentivirus-tumor necrosis factor (TNF)- $\alpha$-RNAi expressing TNF- $\alpha$ small interfering (si)RNA. Using screened siRNA segments, an siRNA was designed. A lentivirus vector expressing siRNA was established and packed as lentivirus particles. A lentivirus that expressed the negative sequence was used as a lentivirus-negative control (NC). The carrageenan-induced SAB model was established in 32 male Sprague-Dawley rats. The modeled rats were randomly assigned to four groups: Lentivirus-RNAi treatment group, lentivirus-NC group, SAB group and phosphate-buffered saline (PBS) blank control group. The lentivirus was injected (1x $10^{7}$ transducing units) into the subacromial bursa of the rats in the lentivirus-RNAi group and lentivirus-NC group, whereas $100 \mu$ l PBS was injected at the same site in the SAB group and the PBS blank control group. At 5 weeks following injection, the animals were sacrificed and venous blood was obtained. The effect of TNF- $\alpha$ interference and the expression of inflammatory cytokines were determined by reverse transcription-quantitative polymerase chain reaction, western blotting, hematoxylin and eosin staining, Van Gieson's staining and immunofluorescence. The expression of TNF- $\alpha$ was decreased in the lentivirus-TNF- $\alpha$-RNAi group compared with that in the SAB group. Morphological observations
\end{abstract}

Correspondence to: Dr Zimin Wang, Department of Orthopaedics, Changhai Hospital, Second Military Medical University, 168 Changhai Road, Shanghai 200433, P.R. China

E-mail: drwangzimin2014@126.com

Key words: lentivirus, rotator cuff disease, tumor necrosis factor- $\alpha$, subacromial bursa, small interfering RNA revealed that the number of inflammatory cells were reduced and damage to tendon fibers was attenuated in this group, suggesting that the downregulation of the protein expression levels of TNF- $\alpha$-associated nuclear factor- $\kappa \mathrm{B}$, matrix metalloproteinase (MMP)1, MMP9, cyclooxygenase (COX)-1 and COX-2 may exert a therapeutic effect on inflammation of the SAB caused by rheumatoid arthritis. It was also found that the expression of stromal cell-derived growth factor-1 was downregulated in the lentivirus-TNF- $\alpha$-RNAi group. Therefore, the present study demonstrated that lentivirus-mediated TNF- $\alpha$ RNAi effectively inhibited the inflammatory response in SAB, and that injection of a lentivirus vector into the affected region is an effective way of achieving RNAi in vivo.

\section{Introduction}

Subacromial bursitis (SAB) is the main cause of pain and dysfunction in shoulder tendinopathy, accounting for $~ 50 \%$ of all cases of shoulder pain (1). The majority of cases of SAB are associated with subacromial impingement syndrome (SIS) and rotator cuff tears. The subacromial bursa lies between the acromion and the rotator cuff, and the friction between them is reduced predominantly by lubrication. Therefore, high levels of exercise are likely to induce inflammation in the shoulder area. Fibrocartilaginous metaplasia in the rotator cuff has previously been reported $(2,3)$. Gotoh et al (4) observed that, compared with unaffected individuals, patients with SIS often presented with a chronic inflammatory response and increased numbers of cluster of differentiation (CD)-2 and CD-11b mononuclear cells in the subacromial bursa. Injection of corticosteroids into the subacromial bursa provided a therapeutic effect, suggesting that SAB occurs as a result of an important pathological change in rotator cuff disease. However, an unavoidable problem in using corticosteroids is their adverse effects. Crofford et al (5) demonstrated that interleukin (IL)-1 and tumor necrosis factor (TNF)- $\alpha$ are able to relieve pain by mediating the expression of cyclooxygenase (COX)-2 and COX-1, suggesting that subacromial bursa cells are important in SAB, by secreting multiple cytokines and forming a network that regulates the chronic inflammatory response, with TNF- $\alpha$ being key in this inflammatory circuit (5). Immunohistochemical studies have reported the 
presence of anti-TNF- $\alpha$ antibody staining in $>80 \%$ of SAB tissue, suggesting that the expression of TNF- $\alpha$ in subacromial bursa cells may also affect the metabolism of these cells, causing damage to the tendon and cartilage (6).

There has been significant interest in using the technique of small interfering RNA (siRNA) to potently suppress genetic expression in a sequence-specific manner (7). In 2003, Song et al (8) demonstrated the therapeutic effect of Fas-specific siRNA in mice with experimentally induced hepatitis. Since then, numerous studies have been performed to investigate the use of siRNA in various disease models (8-12). In the field of locomotor diseases, Schiffelers et al (13) reported that luciferase-specific siRNA reduces luciferase activity in joints. Our previous study also revealed that the fluorescence of the synovium was decreased by green fluorescent protein (GFP)-specific siRNA in the GFP rat (14). These delivery methods for the expression of short hairpin (sh)RNA include the direct application of naked siRNAs and lipid-based delivery vehicles (15). However, the application of these methods is limited by low transduction efficiency, poor control of gene expression and short duration of effect, particularly when it is necessary to generate long-term gene silencing in vivo (16). However, these problems can be addressed using the technique of lentivirus-mediated RNA interference (RNAi) to achieve the desired therapeutic effect (17). To the best of our knowledge, no previous studies have used this technique in the treatment of locomotor diseases, therefore, the present study aimed to use the RNAi technique to silence and inhibit the expression of TNF- $\alpha$ in subacromial bursa cells through local injection of the lentivirus vector specifically targeting TNF- $\alpha$ into the rat subacromial bursa. This may reveal a novel strategy for the gene therapy of SAB.

\section{Materials and methods}

Ethical approval. The present study was approved by the Ethics Committee of the Second Military Medical University (Shanghai, China).

Animals. A total of 32 male Sprague-Dawley rats, aged 3 months and weighing 200-220 g (Shanghai Research Center for Model Organisms, Shanghai, China) were housed under constant temperature $\left(21^{\circ} \mathrm{C}\right)$ and regular light (06:30-19:30 h)-dark (19:30-06:30 h) cycles with ad libitum access to food and water at the Animal Care and Veterinary Services Facility of Fudan University (Shanghai, China), according to the guidelines of the International Council for Laboratory Animal Science. The rats were allowed to acclimate for 2 weeks prior to the initiation of the experiment.

Establishment of the rotator cuff disease model. The rotator cuff disease model was established by subacrominal injection of $10 \mu 13 \%$ carrageenan (Sigma-Aldrich, St. Louis, MO, USA) eight times weekly for 2 weeks as previously described (1). Based on the physiological features of the animals, the injection site was located in the subacromial space above the rotator cuff, to ensure that repeated subacromial saline injections would not damage the tendons (18). Using sterile needles (Microlance 3 , 27G3/4,0.4*19, No.20; Becton-Dickinson, Drogheda, Ireland) and glass syringes (Wegene, Shanghai, China), all injections were made aseptically in the left shoulder under anesthesia with carbon dioxide, according to Soslowsky et al (18). Of the 32 animals, 24 were equally assigned to three groups: A lentivirus TNF- $\alpha$-RNAi group (group A), in which the animals received a local injection of lentivirus-RNAi $1 \times 10^{7}$ tranducing units (TU) per rat in the subacromial bursa; a lentivirus negative control (NC) group (group B), in which the animals received a local injection of lentivirus-NC $1 \times 10^{7} \mathrm{TU}$ per rat in the subacromial bursa, and a rotator cuff disease model group (group C), in which the animals received a local injection of $100 \mathrm{ml}$ phosphate-buffered saline (PBS) per rat. All injections began on day 2 following establishment of the disease model. The eight remaining animals were injected with $10 \mu \mathrm{l}$ PBS as a negative control of the model animals (control).

RNAi design and small interference (siRNA) plasmid construction. siRNAs targeting the TNF- $\alpha$ bases on the pLL3.7 vector (Wegene) were used. The sequence of the siRNAs targeting rat TNF- $\alpha$ were as follows:Rattusnorvegicus; GenBank: L00981.1; TNF- $\alpha, 5$ '-TAATGGCATGGATCTCAAAGATTCAAGAGA TCTTTGAGACCATGCCATTTTTTTTC-3' and 5'-TCGAGA AAAAAAATGGCATGGATCTCAAAGATCTCTTGAATC TTTGAGATCCATGCCATTA-3'. siRNA against rat TNF- $\alpha$ was synthesized by Invitrogen Life Technologies (Carlsbad, CA, USA). The oligos were denatured for $10 \mathrm{~min}$ at $95^{\circ} \mathrm{C}$, using the Applied Biosystems 7500 Real-Time Polymerase Chain Reaction (PCR) system (Applied Biosystems, Foster City, CA, USA) and annealed at room temperature for $>30 \mathrm{~min}$. The paired oligos and pLL3.7 vectors, digested with $\mathrm{XhoI}$ and $\mathrm{HpaI}$, were ligated with $\mathrm{T} 4$ ligase at $16^{\circ} \mathrm{C}$ overnight. Ligation was performed to transform the cells into stable competent cells. The pLL3.7-TNF- $\alpha$-siRNA plasmids were supplied by Shanghai GenePharm Co., Ltd. (Shanghai, China). The plasmid containing TNF- $\alpha$ siRNA was sequenced. A negative control (NC) siRNA containing a scramble sequence was used with the following sequences: 5'-TGCCCTACCACC GAGGTCAATTCAAGAGATTGACCTCGGTGGTAGGGC TTTTTTC-3' and 5-TCGAGAAAAAAGCCCTACCACCGA GGTCAATCTCTTGAATTGACCTCGGTGGTAGGGCA-3'. The TNF- $\alpha$ siRNA-containing plasmid was sequenced and the pLL3.7-TNF- $\alpha$-siRNA was transfected into 293T cells (American Type Culture Collection, Manassas, VA, USA) together with $\Delta 8.9$ and vesicular stomatitis virus glyoprotein (VSVG) (Sigma-Aldrich) to package virus particles with $\mathrm{Ca}^{2+}$ reagent. Briefly, pLL3.7-TNF- $\alpha$-siRNA $(10 \mu \mathrm{g}), \Delta 8.9$ $(8 \mu \mathrm{g})$ and VSVG $(6 \mu \mathrm{g})$ were mixed with $94 \mu \mathrm{l} \mathrm{CaCl} \mathrm{C}_{2}(2 \mathrm{M}$; Sigma-Aldrich), adjusted to $750 \mu \mathrm{l}$ and were added dropwise into $750 \mu \mathrm{l} 2 \mathrm{X}$ Hepes-buffered saline. Following a $5 \mathrm{~min}$ tranquilization step, the mixture was added to the 293T cells pretreated with chloroquine (5 $\mu \mathrm{m}$; Sigma-Aldrich). Following an $8 \mathrm{~h}$ culture period at $37^{\circ} \mathrm{C}$ and a replacement of the medium, the cells were cultured for an additional $48 \mathrm{~h}$ at $37^{\circ} \mathrm{C}$, harvested and centrifuged at $50,000 \mathrm{x}$ g for $120 \mathrm{~min}$ at $10^{\circ} \mathrm{C}$. The supernatant was then discarded. The viral particles were pelleted, titrated by series dilution and then used to infect the 293T cells in the presence of polybrene $8 \mu \mathrm{g} / \mathrm{ml}$ (Sigma-Aldrich). The titer of $3-6 \times 10^{8} \mathrm{TU} / \mathrm{ml}$ was achieved routinely.

Administration of the lentivirus vector in vivo. The animals in group A received a local injection of lentivirus-RNAi 
$1 \times 10^{7} \mathrm{TU} /$ rat in the subacromial bursa and animals in group B received a local injection of lentivirus-NC $1 \times 10^{7} \mathrm{TU} / \mathrm{rat}$ in the subacromial bursa. At 5 weeks following injection, the animals were sacrificed by injection with $4 \%$ pentobarbital sodium (Wegene; $2 \mathrm{ml} / \mathrm{kg}$ ) to obtain the sera and the supra, infraspinatus and subacromial bursa specimens, which were divided into two. The sera and half of the tissue specimens were stored at $-70^{\circ} \mathrm{C}$ and the remaining specimens were fixed in $4 \%$ paraformaldehyde (Sigma-Aldrich) until use.

Histopathological analysis. The supra, infraspinatus and subacromial bursae were fixed in $4 \%$ paraformaldehyde for 2 days, dehydrated, paraffin embedded, sliced parallel to the collagen bundles into $3 \mu \mathrm{m}$ sections and stained with hematoxylin and eosin (HE; Sigma-Aldrich) and Van Gieson's stain (Sigma-Aldrich) for routine histological evaluation. A total of eight $100 \times 100 \mu \mathrm{m}$ visual fields were selected randomly in each section, and the total numbers of inflammatory cells were recorded under a microscope (Leica DMI3000 B, Wetzlar, Germany).

Reverse transcription-quantitative PCR (RT-qPCR) analysis. Total RNA was extracted from $1.0 \mathrm{~g}$ tissue and the neuronal cells using TRIzol ${ }^{\circledR}$ reagent from Invitrogen Life Technologies, according to the manufacturer's instructions. Total RNA was quantified by measuring absorbance at $260 \mathrm{~nm}$ using a Beckman DU640 spectrophotometer (Beckmann Coulter, Fullerton, CA, USA). Reverse transcriptase was used to generate cDNA using $5 \mu \mathrm{g}$ RNA and oligo(dT) primers, according to manufacturer's instructions (Invitrogen Life Technologies). The reactions were run on a Real-Time PCR system (Applied Biosystems 7500 PCR) with the following cycle conditions: $95^{\circ} \mathrm{C}$ for $15 \mathrm{sec}, 45$ cycles at $95^{\circ} \mathrm{C}$ for $5 \mathrm{sec}$ and at $60^{\circ} \mathrm{C}$ for $30 \mathrm{sec}$. A standard curve for TNF- $\alpha$ was generated by using serially diluted total RNA from neuronal cells and the spinal cord to quantitate relative mRNA levels of TNF- $\alpha$. The sequences of the TNF- $\alpha$ gene primers (Sangon Biotechnology Co., Ltd., Shanghai, China) were as follows: Forward 5'-TGTCTGTGCCTCAGCCTCTTC-3' and reverse 5'-TTTGGGGAACTTCTCCTCCTTG-3', with a product of $110 \mathrm{bp}$. GAPDH served as a normalization control. The primers sequences for GAPDH were as follows: Forward 5'-TGGAGAAACCTGCCAAGTATGA'-3 and reverse 5'-TGG AAGAATGGGAGTTGCTGT-3', with a product of $135 \mathrm{bp}$. The relative expression of mRNA was calculated using the $2^{-\Delta \Delta C t}$ method (19).

Western blotting. The tissues were washed with cold PBS containing 2 mM EDTA (Sigma-Aldrich) and lysed with denaturing SDS-PAGE sample buffer (United States Biological, Salem, MA, USA) using standard methods. Briefly, the cell pellets were resuspended in $100 \mu$ lysis buffer $(20 \mathrm{mM}$ Tris. $\mathrm{Cl} \mathrm{pH} 7.9,1 \mathrm{mM}$ EDTA, 5\% Glycerol) with an equal volume of $0.5 \mathrm{~mm}$ glass beads (Biospec products, Inc., Bartlesville, OK, USA) and vortexed for $10 \mathrm{~min}$ at $4^{\circ} \mathrm{C}$. The total protein concentration was determined using a protein assay kit (Thermo Fisher Scientific) and analyzed with AlphaView SA software (Cell Biosciences, Inc., San Jose, CA, USA). The final concentration of protein in each sample was adjusted to $2 \mathrm{mg} / \mathrm{ml}$. The protein lysates were separated by

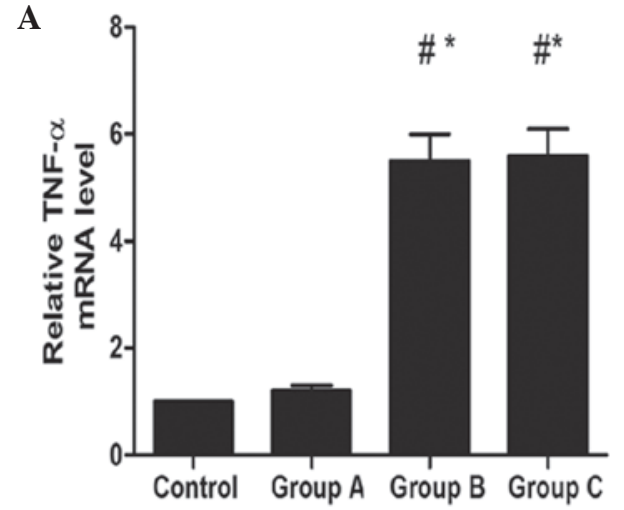

B Control Group A Group B Group C

TNFa

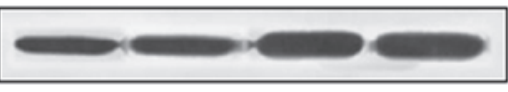

GADPH

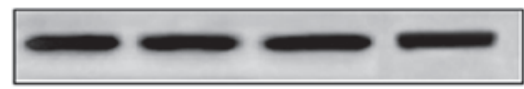

C

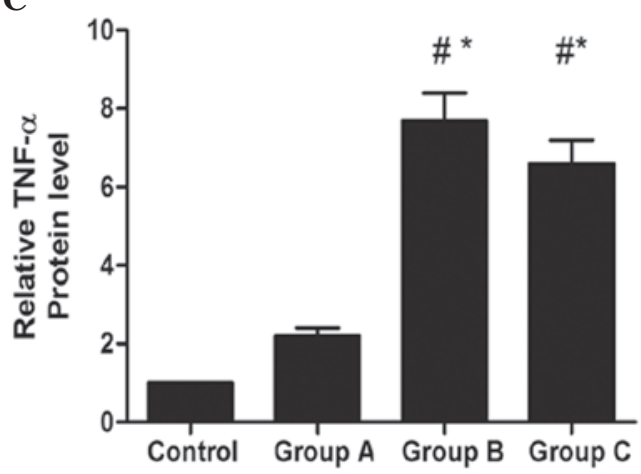

Figure 1. Lentivirus-mediated TNF- $\alpha$ siRNA inhibits the expression of TNF- $\alpha$ markedly. (A) Reverse transcription-quantitative polymerase chain reaction demonstrating marked inhibition of the mRNA expression of TNF- $\alpha$ by siRNA in the subacromial bursa. (B and C) Densitometric analysis revealed the expression of TNF- $\alpha$ under different treatment conditions. Each column represents pooled data $(n=4)$. The vertical bars represent the mean \pm standard error of the mean. ${ }^{~} \mathrm{P}<0.05$, compared with the control, ${ }^{*} \mathrm{P}<0.05$ compared with group $\mathrm{A}$. Group $\mathrm{A}$, lentivirus-TNF- $\alpha-\mathrm{RNAi}$ injection; group $\mathrm{B}$, lentivirus-NC injection; group $\mathrm{C}$, phosphate-buffered saline injection. TNF, tumor necrosis factor; siRNA, short interfering RNA.

$12 \%$ SDS-PAGE and transferred onto polyvinylidene fluoride membranes (Millipore, Bedford, MA, USA), incubated with primary antibodies and blocked with $5 \%$ bovine serum albumin (BSA; Sigma-Aldrich). The primary antibodies $(1: 1,000)$ used were TNF- $\alpha$ (cat. no. bs-2081R; Bioss, Woburn, MA , USA), NF-кB (cat. no. 4790; Cell Signaling Technology, Danvers, MA, USA), matrix metalloproteinase (MMP)-1 (cat. no. MAB13439; Millipore), MMP-9 (cat. no. BS-1241; Bioworld, St Louis Park, MN, USA), COX-1 (cat. no. BS1075; Bioworld), COX-2 (cat. no. MAB3462; Millipore), and stromal cell-derived growth factor-1 (SDF-1; cat. no. 3740S; Cell Signaling Technology). The membranes were washed with Tris-buffered saline (Sigma-Aldrich) three times and 

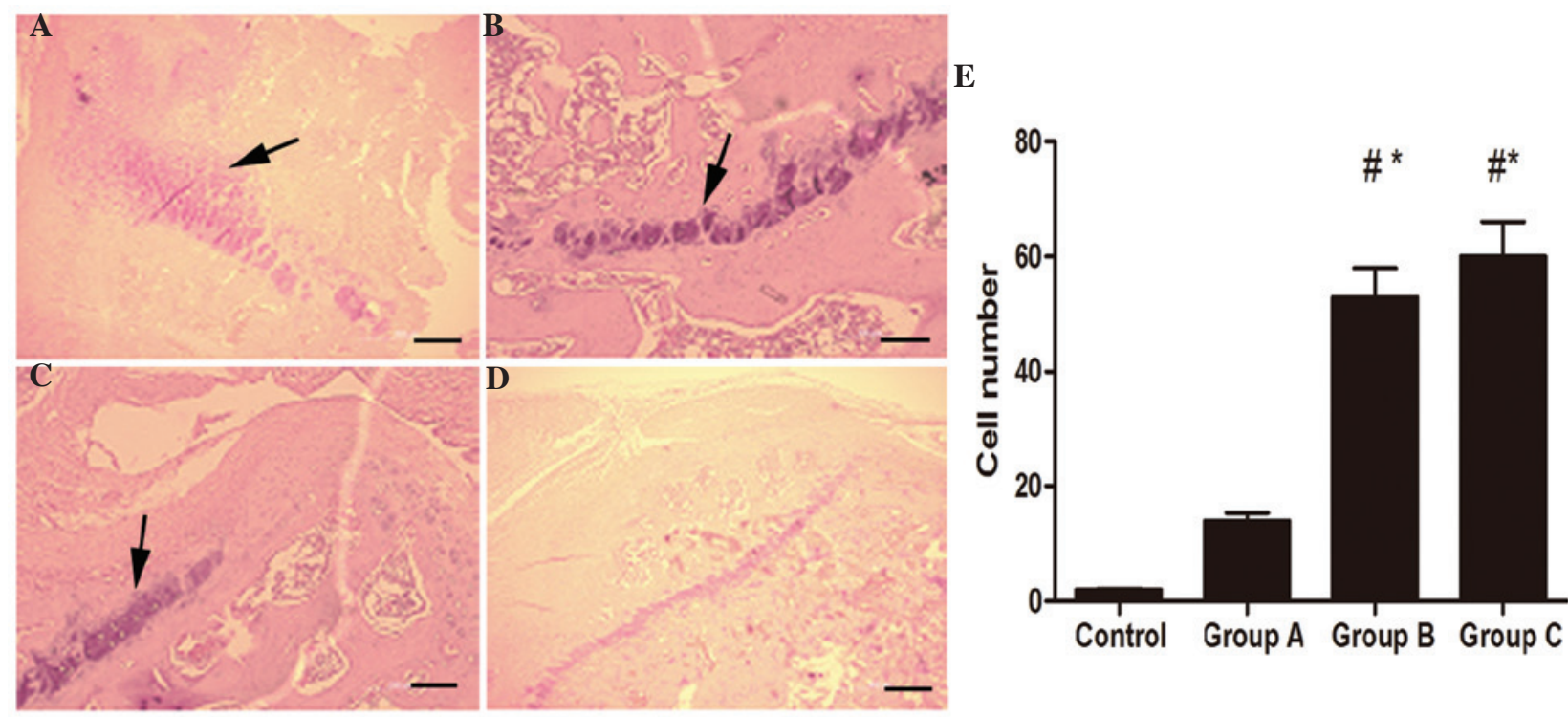

Figure 2. (A) Histopathological images of hippocampal tissues from rats in the different groups. (A) Low levels of inflammatory cell infiltration were observed in groups A. (B and C) Relatively large quantities of inflammatory cells were observed in groups B and C. (D) No inflammatory cells were observed in the tendon in the control group. Arrows indicate the inflammatory cells (hematoxylin and eosin; magnification, x200). Scale bar, $100 \mu \mathrm{m}$. (E) Number of cells per visual field in each group following injection. All data are presented as the mean \pm standard error of the mean in four rats. ${ }^{. P}<0.05$, compared with the control; ${ }^{*} \mathrm{P}<0.05$ compared with group A. Group A, lentivirus-TNF- $\alpha$-RNAi injection; group B, lentivirus-NC injection; group C phosphate-buffered saline injection.

incubated with horseradish peroxidase (HRP)-conjugated goat anti-rabbit immunoglobulin G antibody (1:5,000; Santa Cruz Biotechnology, Inc., Santa Cruz, CA, USA) at room temperature for $2 \mathrm{~h}$. Signal detection was performed using an enhanced chemiluminescence system (GE Healthcare, Waukesha, WI, USA). The protein levels in the tissues were quantified by densitometry and normalized to GAPDH, with the levels of the phospho-Ras homolog gene family, member A (RhoA) normalized to the total levels of RhoA.

Immunohistochemistry. The rat tissue sections $(5 \mu \mathrm{m})$ were treated with 0.1 M PB:methanol (1:1) (Sigma-Aldrich) and $1 \%$ hydrogen peroxide (Sigma-Aldrich) for $20 \mathrm{~min}$, followed by incubation with $0.1 \mathrm{M}$ phosphate buffer (PB) with $1 \%$ BSA and $0.3 \%$ Triton X-100 (Wegene) for $1 \mathrm{~h}$. Subsequently, the sections were incubated overnight at $4^{\circ} \mathrm{C}$ with mouse anti-rat-fibronectin primary antibody (Santa Cruz Biotechnology, Inc.) in $0.1 \mathrm{M}$ PB with $0.3 \%$ BSA and $0.3 \%$ Triton X-100, followed by a biotinylated mouse secondary antibody and the Avidin-biotin Complex-HRP kit (Thermo Fisher Scientific, Inc., Wilmington, MA, USA) for $2 \mathrm{~h}$. Specific binding was visualized with diaminobenzidine (Santa Cruz Biotechnology, Inc.). The tissue was then dehydrated, covered with mounting solution and observed under an Eclipse E200 optical microscope (Nikon, Tokyo, Japan).

ELISA. The serum protein level of TNF- $\alpha$ was determined using an ELISA kit (R\&D Systems, Minneapolis, MN, USA), according to the manufacturer's instructions. The results were normalized to the number of rats per group. The data are presented as mean \pm standard error of the mean from four independent experiments, which were performed in triplicate.

Statistical analysis. The parametric data were compared using multi-variable two-way analysis of variance followed by
Tukey's post hoc test for comparison between several independent groups. $\mathrm{P}<0.05$ was considered to indicate a statistically significant difference in a two-tailed test. Comparisons between two groups were performed using Student's t-test. Data are expressed as the mean \pm standard error of the mean. Data were analyzed using SPSS version 16.0 statistical software (SPSS, Inc., Chicago, IL, USA).

\section{Results}

Gene-silencing effects of siRNA in vivo. At 5 weeks following the injection of siTNF- $\alpha$, the levels of mRNA from the tendon of animals in the the rotator cuff disease model were determined by RT-qPCR to compare the in vivo gene-silencing effect of TNF- $\alpha$ among groups A, B and C. The expression of TNF- $\alpha$ in the subacromial bursa of group A was reduced compared with the expression levels in groups B and C (Fig. 1A). Western blot analysis revealed that the protein expression of TNF- $\alpha$ in group A was significantly decreased compared with the levels observed in groups B and C (Fig. 1B and C).

Histopathological evaluation. In the animals in the control group, which received normal saline, no inflammatory cells were observed in the subacromial space. Inflammatory cell infiltration was observed in $12.5 \pm 9.8$ cells/visual field in group A vs. $53.2 \pm 8.7$ and $57.8 \pm 8.9$ cells/visual field in groups $\mathrm{B}$ and $\mathrm{C}$. The inflammatory response was predominantly characterized by infiltration of relatively large quantities of lymphocytes and several neutrophils on the tendon surface (Fig. 2).

Microscopic evaluation of the tendon. Fibronectin was analyzed in order to detect signs of an inflammatory response (Fig. 3). A significant difference was identified in 


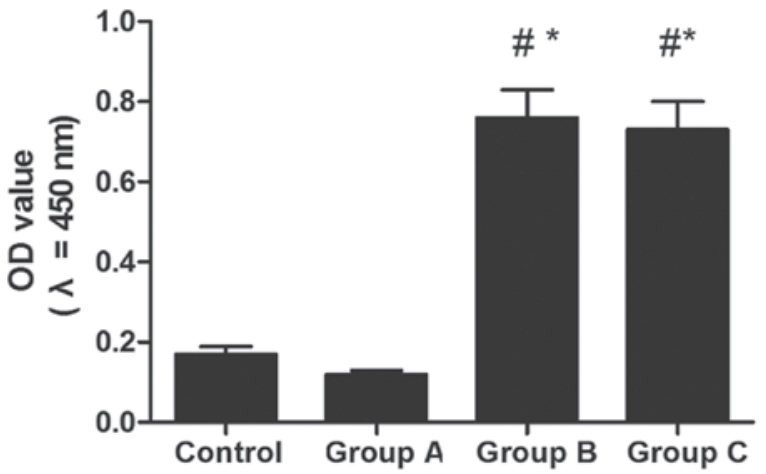

Figure 3. Expression of fibronectin. A significant difference was observed in the OD of fibronectin between groups A, B and C $(\mathrm{P}<0.001)$. Data are presented as the mean \pm standard error of the mean in four rats. ${ }^{~} \mathrm{P}<0.05$, compared with the control group; ${ }^{*} \mathrm{P}<0.05$, compared with group $\mathrm{A}$. Control, untreated; Group A, lentivirus-TNF- $\alpha-R N A i$ injection; group B, lentivirus-NC injection; group C phosphate-buffered saline injection; OD, optical density.

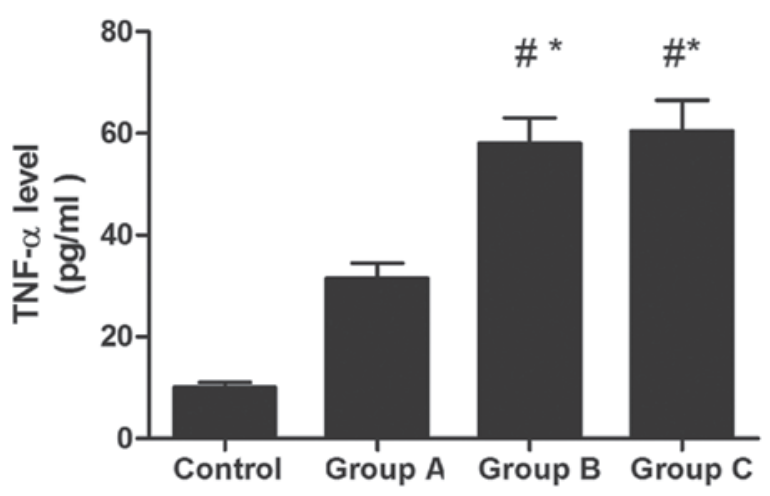

Figure 4. Serum levels of TNF- $\alpha$ following lentivirus-mediated RNA interference. All data are presented as the mean \pm standard error of the mean in four rats. ${ }^{*} \mathrm{P}<0.05$, compared with the control; ${ }^{*} \mathrm{P}<0.05$ compared with group A. Control, untreated; Group A, lentivirus-TNF- $\alpha$-RNAi injection; group $\mathrm{B}$, lentivirus-NC injection; group $\mathrm{C}$ phosphate-buffered saline injection; TNF, tumor necrosis factor.

the optical densities of the expression of fibronectin among groups $\mathrm{A}, \mathrm{B}$ and $\mathrm{C}(\mathrm{P}<0.001)$.

Levels of TNF- $\alpha$ in the serum following lentivirus-mediated RNAi treatment. At 5 weeks subsequent to treatment with the lentivirus-mediated RNAi, the level of TNF- $\alpha$ in the serum was decreased significantly in group $\mathrm{A}$, as compared with that in groups B and C (Fig. 4).

TNF- $\alpha$ RNAi modulates the expression levels of $N F-\kappa B$, MMP-1, MMP-9, COX-1, COX-2 and SDF-1 in rotator cuff disease model rats. The effects of TNF- $\alpha$ silencing on the expression levels of NF- $\mathrm{B}, \mathrm{MMP}-1, \mathrm{MMP}-9, \mathrm{COX}-1$, COX-2 and SDF-1 in vivo were determined by injecting lentivirus-siTNF- $\alpha$ into the rat subacromial bursa. At 5 weeks following injection, the animals were sacrificed via the injection of $4 \%$ pentobarbital sodium $2 \mathrm{ml} / \mathrm{kg}$. The tendons were dissected and homogenized to extract proteins for western blot analysis, to determine the protein expression levels of $\mathrm{NF}-\kappa \mathrm{B}$, MMP-1, MMP-9, COX-1, COX-2 and SDF-1. The expression levels of NF- $\mathrm{B}$, MMP-1, MMP-9, COX-1, COX-2 and SDF-1
A
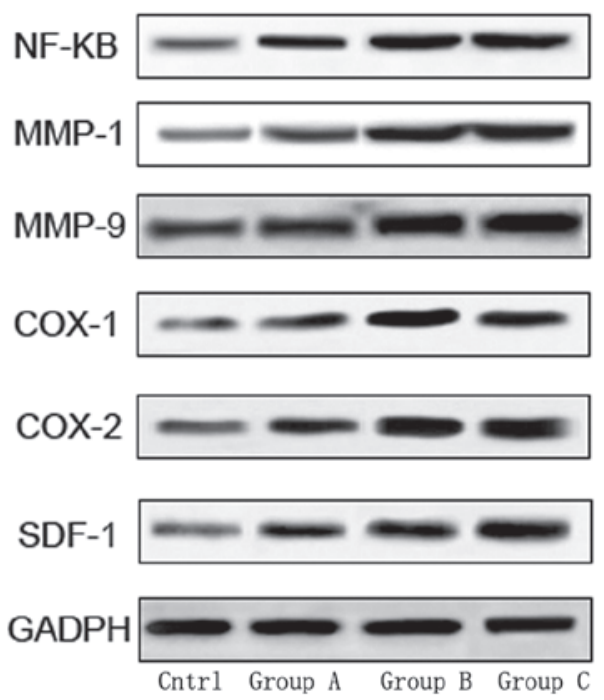

B

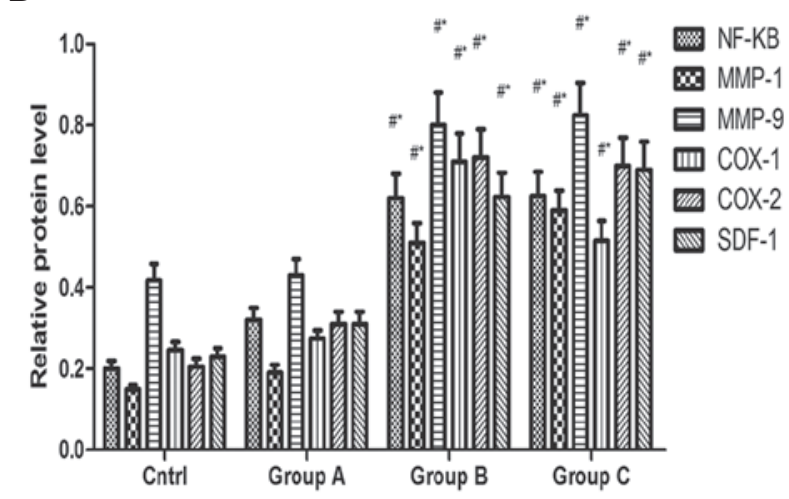

Figure 5. (A) Expression levels of NF-кB, MMP-1, MMP-9, COX-1, COX-2 and SDF-1 following TNF- $\alpha$ expression silencing in groups A, B and C. GAPDH indicates equal loading of protein. (B) Densitometric analysis of the expression levels of NF- $\mathrm{kB}$, MMP-1, MMP-9, COX-1, COX-2 and SDF-1 in the different treatment groups. Each column represents pooled data $(n=4)$. The vertical bars represent the mean \pm standard error of the mean. ${ }^{~} \mathrm{P}<0.05$, compared with the control; " $\mathrm{P}<0.05$ compared with group A. Group A, lentivirus-TNF- $\alpha$-RNAi injection; group $B$, lentivirus-NC injection; group $C$ phosphate-buffered saline injection; NF, nuclear factor; MMP, matrix metalloproteinase; COX, cyclooxygenase; SDF, stromal cell-derived factor; TNF, tumor necrosis factor.

in group B were significantly increased compared with those in group $\mathrm{C}$ and the control group, while they were significantly decreased in group A compared with groups B and C (Fig. 5).

\section{Discussion}

As TNF- $\alpha$ is in the master regulator of the inflammatory response in $\mathrm{SAB}$, its expression level directly affects the expression of the other inflammatory cytokines. Whether is it possible to attenuate the increased generation of inflammatory cytokines by inhibiting the expression of TNF- $\alpha$, to limit the progression of inflammation-induced injuries remains to be elucidated To investigate this, the present study used a lentivirus as a vector to silence TNF- $\alpha$, which was then injected into the left subacromial bursa in a carrageenan-induced rat SAB model. RT-qPCR and western blot analyses revealed 
that the expression of TNF- $\alpha$ in the muscle and bursal cavity surrounding the injection site was decreased significantly 5 weeks after injection, and the inflammatory response in the tendon was effectively downregulated, attenuating the fibrocartilaginous metaplasia in the rotator cuff.

Voloshin et al (6) demonstrated that TNF- $\alpha$ is an important factor, which mediates the occurrence of inflammation and pain. It is a gene, which initiates transcription and is located upstream of tissue metalloproteinase and COX. Kim et al (17) demonstrated that SAB cells secrete a large quantity of SDF-1, ten times higher than that of normal bursal cells. Yanagisawa et al (20) revealed that the increased level of vascular endothelial growth factor in the subacromial bursa of patients with rotator cuff tear is closely correlated with the inflammatory response. In a study on changes in the gene expression profile of SAB using the gene chip technique and immunohistochemistry, Blaine et al (21) found that the expression levels of IL-1 $\alpha$, IL-1 $\beta$, IL-6 and TNF- $\alpha$ in inflammatory cytokines, MMP-1 and MMP-9 in tissue metalloproteinase, and COX-1 and COX-2 in cyclooxygenase increase markedly, and that SAB bursal cells are the predominant source of secretion of these factors. It has been observed that the expression of IL-1 is positively correlated with bursal inflammation and shoulder joint pain in SAB (6). Numerous studies on arthropathies have demonstrated that $\mathrm{NF}-\kappa \mathrm{B}$ is also an important transcription factor involved in the expression of inflammatory cytokines (22). Schaffner et al (23) observed that arginine-glycine-aspartic acid polypeptide induces synovial fibroblasts to express MMP-1, indicating that the induction of expression of MMP by the central cell binding domain fibronectin may be mediated by $\alpha 5 \beta 1$. The NF- $\mathrm{BB}$ signaling transduction pathway is the common intracellular signaling transduction pathway of TNF- $\alpha$, IL- $1 \beta$ and other inflammatory mediators. NF- $\kappa \mathrm{B}$ activation may increase the transcription levels of IL-1B, IL-6, IL-8, TNF- $\alpha$, MMP, adhesion molecules and cyclooxygenase genes (22). It was revealed in the present study that serum levels of TNF- $\alpha$ were increased in groups B and C. TNF- $\alpha$ has inflammation-mediatory and immunoregulatory roles in the immune response. Its effects include activating lymphocytes and releasing other inflammatory cytokines, including IL-1 and IL-6, prostaglandin and metalloproteinase $(24,25)$. As TNF- $\alpha$ also promotes angiogenesis and regulates adhesion molecules, it is an important reactivator in the inflammatory response (26).

In the present study, it was observed that the protein expression of NF- $\kappa \mathrm{B}$ was downregulated following TNF- $\alpha$ interference, which further decreased the protein expression levels of MMP-1, MMP-9, COX-1 and COX-2. This finding fully supported the hypothesis of TNF- $\alpha$ as an important mediator of inflammation and pain, as well as an upstream gene of tissue metalloproteinase and cyclooxygenase. It is noteworthy that SDF-1 decreased with TNF- $\alpha$ gene knockout, indicating that TNF- $\alpha$ also has a positive feedback effect on SDF-1 in the SAB network, which was consistent with previous studies (1). The immunohistochemical staining results demonstrated that the inflammation-induced decrease of fibronectin was attenuated following TNF- $\alpha$ gene knockout, indicating the TNF- $\alpha$ gene knockout effectively reduced inflammation-induced injuries.
The active TNF- $\alpha$ siRNA sequence was used to construct a siRNA expression cassette, which was then incorporated into a lentiviral vector system. A key advantage of lentiviral vectors over other gene delivery systems is that they are able to efficiently transduce post-mitotic cells, including subacromial bursa cells, as lentiviral vectors enable the transduction of non-dividing cells and can result in long-term gene expression in subacromial bursa cells.

In conclusion, the present study succeeded in knocking out the mRNA expression of TNF- $\alpha$ and downregulating the protei expression levels of MMP-1, MMP-9, COX-1, COX-2 and SDF-1 in the inflammatory network by injecting siRNA TNF- $\alpha$ into the subacromial bursa of patients with SAB. This suggested that use of the lentivirus-mediated RNAi technique to regulate the key target in the chronic SAB inflammatory response circuit may prove to be an effective approach for the clinical treatment of SAB or rotator cuff disease in the future.

\section{Acknowledgements}

The present study was supported by grants from the National Natural Science Foundation of China (grant no. 81171766), and the Foundation of Science and Technology Commission of Shanghai (grant no. 08QA1400400).

\section{References}

1. Blaine TA, Cote MA, Proto A, Mulcahey M, Lee FY and Bigliani LU: Interleukin-1beta stimulates stromal-derived factor-1alpha expression in human subacromial bursa. J Orthop Res 29: 1695-1699, 2011.

2. Chard MD, Cawston TE, Riley GP, Gresham GA and Hazleman BL: Rotator cuff degeneration and lateral epicondylitis: a comparative histological study. Ann Rheum Dis 53: 30-34, 1994.

3. Fukuda H, Hamada K and Yamanaka K: Pathology and pathogenesis of bursal-side rotator cuff tears viewed from en bloc histologic sections. Clin Orthop Relat Res 254: 75-80, 1990.

4. Gotoh M, Hamada K, Yamakawa H, et al: Interleukin-1-induced subacromial synovitis and shoulder pain in rotator cuff diseases. Rheumatology (Oxford) 40: 995-1001, 2001.

5. Crofford LJ, Tan B, McCarthy CJ and Hla T: Involvement of nuclear factor kappa $\mathrm{B}$ in the regulation of cyclooxygenase-2 expression by interleukin-1 in rheumatoid synoviocytes. Arthritis Rheum 40: 226-236, 1997.

6. Voloshin I, Gelinas J, Maloney MD, O'Keefe RJ, Bigliani LU and Blaine TA: Proinflammatory cytokines and metalloproteases are expressed in the subacromial bursa in patients with rotator cuff disease. Arthroscopy 21: 1076.e1-1076.e9, 2005.

7. Elbashir SM, Harborth J, Lendeckel W, Yalcin A, Weber K and Tuschl T: Duplexes of 21-nucleotide RNAs mediate RNA interference in cultured mammalian cells. Nature 411: 494-498, 2001.

8. Song E, Lee SK, Wang J, et al: RNA interference targeting Fas protects mice from fulminant hepatitis. Nat Med 9: 347-351, 2003.

9. Verma UN, Surabhi RM,Schmaltieg A, Becerra C and Gaynor RB: Small interfering RNAs directed against beta-catenin inhibit the in vitro and in vivo growth of colon cancer cells. Clin Cancer Res 9: 1291-1300, 2003.

10. Takei Y, Kadomatsu K, Yuzawa Y, Matsuo S and Muramatsu T: A small interfering RNA targeting vascular endothelial growth factor as cancer therapeutics. Cancer Res 64: 3365-3370, 2004.

11. Aharinejad S, Paulus P, Sioud M, et al: Colony-stimulating factor-1 blockade by antisense oligonucleotides and small interfering RNAs suppresses growth of human mammary tumor xenografts in mice. Cancer Res 64: 5378-5384, 2004.

12. Zhang W, Yang H, Kong X, et al: Inhibition of respiratory syncytial virus infection with intranasal siRNA nanoparticles targeting the viral NS1 gene. Nat Med 11: 56-62, 2005. 
13. Schiffelers RM, Xu J, Storm G, Woodle MC and Scaria PV: Effects of treatment with small interfering RNA on joint inflammation in mice with collagen-induced arthritis. Arthritis Rheum 52: 1314-1318, 2005.

14. Sui J and Wang ZM: Value of bursectomy in the surgical treatments for shoulder impingement syndrome. Zhong Guo Gu Ke Za Zhi 21: 37-40, 2013 (In Chinese).

15. Luo MC, Zhang DQ, Ma SW, et al: An efficient intrathecal delivery of small interfering RNA to the spinal cord and peripheral neurons. Mol Pain 1: 29, 2005.

16. Hassani Z, Lemkine GF, Erbacher P, et al: Lipid-mediated siRNA delivery down-regulates exogenous gene expression in the mouse brain at picomolar levels. J Gene Med 7: 198-207, 2005.

17. Kim EY,Hong YB, Lai Z, et al: Expression and secretion of human glucocerebrosidase mediated by recombinant lentivirus vectors in vitro and in vivo: implications for gene therapy of Gaucher disease. Biochem Biophys Res Commun 318: 381-390, 2004.

18. Soslowsky LJ, Carpenter JE, DeBano CM, Banerji I and Moalli MR: Development and use of an animal model for investigations on rotator cuff disease. J Shoulder Elbow Surg 5: 383-392, 1996.

19. Figuiredo A, Cordeiro AL, Tomada N, Tomada I, Rodrigues A, Gouveia A and Neves D: Real-time PCR study of Ang1, Ang2, Tie-2, VEGF, and KDR expression in human erectile tissue during aging. J Sex Med 8: 1341-1351, 2011.
20. Yanagisawa K, Hamada K, Gotoh M, et al: Vascular endothelial growth factor (VEGF) expression in the subacromial bursa is increased in patients with impingement syndrome. J Orthop Res 19: 448-455, 2001.

21. Blaine TA, Kim YS, Voloshin I, et al: The molecular pathophysiology of subacromial bursitis in rotator cuff disease. J Shoulder Elbow 14 (Suppl): 84-89, 2005.

22. Thiele K, Bierhaus A, Autschbach F, et al: Cell specific effects of glucocorticoid treatment on the NF-kappaBp65/IkappaBalpha system in patients with Crohn's disease. Gut 45: 693-704, 1999.

23. Schaffner F1, Ray AM and Dontenwill M: Integrin $\alpha 5 \beta 1$, the Fibronectin Receptor, as a Pertinent Therapeutic Target in Solid Tumors. Cancers (Basel) 5: 27-47, 2013.

24. Ernandez T and Mayadas T: Immunoregulatory role of TNFalpha in inflammatory kidney diseases. Kidney Int 76: 262-276, 2009.

25. Thomas PS: Tumour necrosis factor-alpha: The role of this multifunctional cytokine in asthma. Immunol Cell Biol 79: 132-140, 2001

26. Foxwell BM, Bondeson J, Brennan F and Feldmann M: Adenoviral transgene delivery provides an approach to identifying important molecular processes in inflammation: evidence for heterogenecity in the requirement for NFkappaB in tumour necrosis factor production. Ann Rheum Dis 59: i54-i59, 2000. 